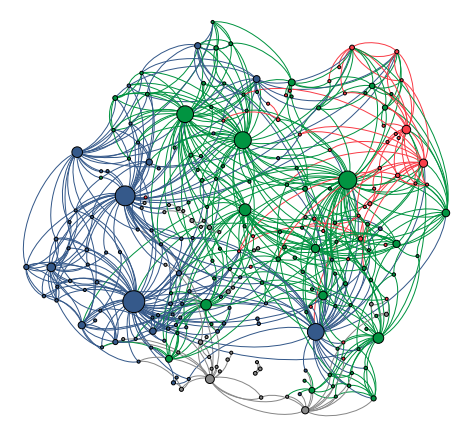

Figure 6: Recommendation graph of YouTube channels.

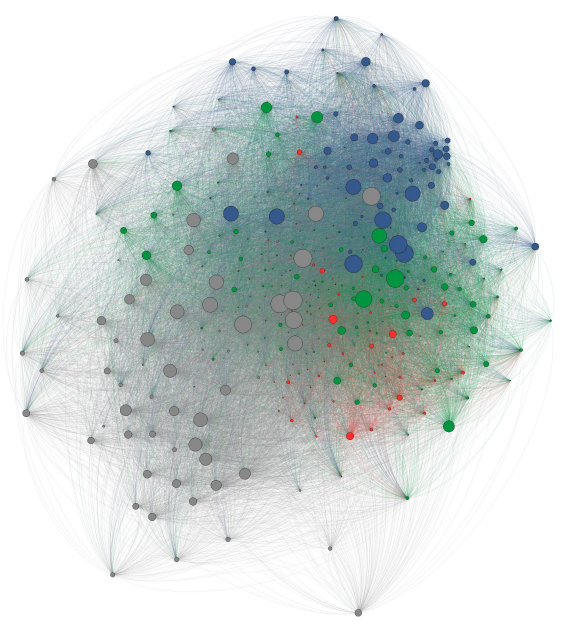

Figure 7: Recommendation graph of YouTube videos. Colors for communities are the same as those in the paper.

\section{A DATA COLLECTION}

We give some details in the data collection process. Tab. 7 and Tab. 8 show for channels labeled as Alt-right, Alt-lite and I.D.W., their communities and data collection steps. Tab 9 shows all media channels we obtained. Fig. 9 highlights what was collected on YouTube. Below, we enumerate the keywords employed to search for channels of each of the communities:

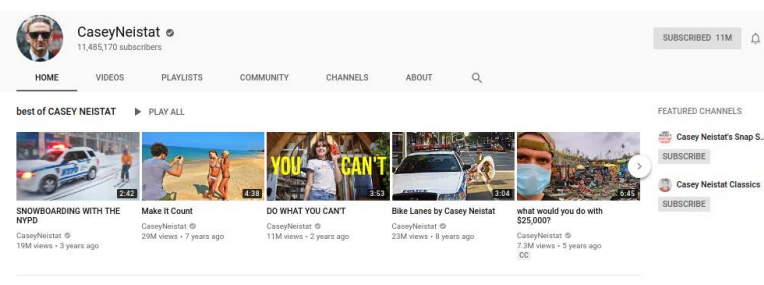

Figure 8: Example of YouTube channel with featured channel on the side.

For the I.D.W. Stephen Hicks, Camille Paglia, Carl Benjamin, Elon Musk, Akira the Don, Nicholas Christakis, Claire Lehmann, Matt Christiansen, Steven Pinker, Rebel Wisdom, Tim Pool, Quillette, fonathan Haidt, Peter Thiel, Lindsay Shepherd, James Damore

For the Alt-lite Brittany Pettibone, Jack Posobiec, Gavin McInnes, Kyle Chapman, Kyle Prescott, Lucian Wintrich, Mike Cernovich, Milo Yiannopoulos, Stefan Molyneaux, Vee, Blonde in the Belly of the Beast, Paul foseph Watson, Styxhenxenhammer666, Rebel Media, Lauren Chen, Computing Forever, Andy Warski, Owen Benjamin, Steven Crowder

For the Alt-right Evola, Evropa, The fewish Question, White Genocide, Mass immigration, Andrew Anglin, weev, Andy Nowicki, Augustus Invictus, Christopher Cantwell, Collin Liddell, Daniel f. Kleve, Daniel Friberg, Dillon Irizarry, Greg Johnson, Fared Taylor, fason Kessler, Jason Reza Jorjani, Johnny Monoxide, Lana Lokteff, Matt Forney, Matthew Heimbach, Matthew Parrott, Mike Enoch, Nathan Damigo, Pax Dickinson, Richard Spencer, Tara McCarthy, Vox Day, Baked Alaska

\section{B FEATURED VS RECOMMENDED}

We illustrate the difference between featured and recommended channel. In Fig. 8 you may see an example of featured channels, these are chosen by the channel owner. In Fig. 9, letter (e) shows related channels, these are recommendations made by YouTube.

\section{LIKES, VIDEOS, VIEWS, COMMENTS}

Tab. 4 shows, for the three communities, the number of likes, views, videos and commenting users across the years.

\section{USER TRAJECTORIES}

Tab. 5 shows the absolute numbers of users tracked and infected (at all levels, as mentioned in Sec. 6. It also shows what percentage of the total number of users who watched Alt-right the number of users infected was. Additionally, In Tab. 6, we show the trajectories from the Alt-right to the other two communities and media channel (that is, we repeat the exact same procedure tracking users from the Alt-right and checking if they commented in the other communities). We find that users from the Alt-right diffuse in similar propotions to the other communities and the media channels.

\section{E RECOMMENDATION GRAPHS}

In Figs. 6 and 7 we show the recommendation graphs used for the experiment in Section 7. 
Table 4: For all categories, we list the number of likes, views, videos and commenting users across the years.

\begin{tabular}{|c|c|c|c|c|c|}
\hline Category & Year & Like Count & View Count & Video Count & Comment Count \\
\hline \multirow[t]{11}{*}{ Alt-lite } & 2008 & 272639 & 18145720 & 1392 & 129130 \\
\hline & 2009 & 585060 & 32993863 & 929 & 197934 \\
\hline & 2010 & 503744 & 30519109 & 1498 & 248432 \\
\hline & 2011 & 527221 & 28400257 & 2344 & 236961 \\
\hline & 2012 & 805166 & 61779929 & 4142 & 360688 \\
\hline & 2013 & 1237131 & 101136564 & 2476 & 531614 \\
\hline & 2014 & 2574586 & 158822748 & 3319 & 824757 \\
\hline & 2015 & 8227303 & 398745164 & 7299 & 2787437 \\
\hline & 2016 & 27277364 & 1001985084 & 9442 & 8983525 \\
\hline & 2017 & 55014745 & 1393845365 & 15858 & 12322105 \\
\hline & 2018 & 54723719 & 1092143577 & 20681 & 21341673 \\
\hline \multirow{11}{*}{ Alt-right } & 2008 & 559 & 73159 & 29 & 332 \\
\hline & 2009 & 8389 & 1236895 & 313 & 1135 \\
\hline & 2010 & 14124 & 1897892 & 363 & 2136 \\
\hline & 2011 & 15992 & 1778120 & 174 & 6076 \\
\hline & 2012 & 75092 & 4925868 & 434 & 10452 \\
\hline & 2013 & 160494 & 11219639 & 654 & 25825 \\
\hline & 2014 & 233381 & 12718956 & 725 & 47032 \\
\hline & 2015 & 434925 & 17148672 & 958 & 127319 \\
\hline & 2016 & 1411778 & 44177307 & 2334 & 529821 \\
\hline & 2017 & 4253888 & 108482909 & 3548 & 1262549 \\
\hline & 2018 & 5773031 & 106455102 & 5843 & 2725573 \\
\hline \multirow[t]{11}{*}{ Media } & 2008 & 348137 & 128986765 & 2115 & 7932 \\
\hline & 2009 & 511468 & 196992273 & 3939 & 41492 \\
\hline & 2010 & 573299 & 203399250 & 6531 & 94379 \\
\hline & 2011 & 1824078 & 350120542 & 12748 & 200385 \\
\hline & 2012 & 3432239 & 454969357 & 25716 & 447302 \\
\hline & 2013 & 5238196 & 716009326 & 18135 & 756691 \\
\hline & 2014 & 9217725 & 1538251895 & 18836 & 814124 \\
\hline & 2015 & 16569182 & 2015671151 & 24168 & 830655 \\
\hline & 2016 & 27807514 & 2481994316 & 30119 & 1317648 \\
\hline & 2017 & 46467022 & 3102590498 & 35678 & 2931209 \\
\hline & 2018 & 54106314 & 2997876294 & 30951 & 13667470 \\
\hline \multirow[t]{11}{*}{ I.D.W. } & 2008 & 54185 & 7034287 & 447 & 5263 \\
\hline & 2009 & 61340 & 8661426 & 488 & 11249 \\
\hline & 2010 & 135205 & 15457288 & 549 & 29932 \\
\hline & 2011 & 269021 & 22797055 & 884 & 129453 \\
\hline & 2012 & 365241 & 23918023 & 1067 & 154322 \\
\hline & 2013 & 1085086 & 109350887 & 2520 & 226833 \\
\hline & 2014 & 2451712 & 230853763 & 2304 & 367374 \\
\hline & 2015 & 3297678 & 261930446 & 2053 & 858816 \\
\hline & 2016 & 6616069 & 447000398 & 3291 & 2056423 \\
\hline & 2017 & 18820727 & 1135173733 & 8789 & 4291180 \\
\hline & 2018 & 25625709 & 1575685392 & 14842 & 11013421 \\
\hline
\end{tabular}


Table 5: We show absolute numbers for users infected and tracked in Sec. 6, as well as what percentage of the total number of users who watched Alt-right the number of users infected was.

\begin{tabular}{|c|c|c|c|c|c|c|}
\hline Category & Start & Year & \# Users Infected & \# Users Tracked & $\%$ of Users Alt-right & $\%$ Tracked and Infected \\
\hline \multirow[t]{14}{*}{ Alt-lite } & \multirow[t]{5}{*}{ 2006-2012 } & 2006-2012 & 0 & 170301 & $0 \%$ & $0 \%$ \\
\hline & & 2013-2016 & 2132 & 43872 & $146 \%$ & $4.86 \%$ \\
\hline & & 2016 & 3426 & 27045 & $8.83 \%$ & $12.67 \%$ \\
\hline & & 2017 & 4558 & 28944 & $6.1 \%$ & $15.75 \%$ \\
\hline & & 2018 & 6186 & 34436 & $4.37 \%$ & $17.96 \%$ \\
\hline & \multirow[t]{4}{*}{$2013-2016$} & 2013-2016 & 0 & 414353 & $0 \%$ & $0 \%$ \\
\hline & & 2016 & 12287 & 127591 & $25.8 \%$ & $9.63 \%$ \\
\hline & & 2017 & 16345 & 117181 & $19.71 \%$ & $13.95 \%$ \\
\hline & & 2018 & 22753 & 126815 & $15.39 \%$ & $17.94 \%$ \\
\hline & \multirow{3}{*}{2016} & 2016 & 0 & 718464 & $0 \%$ & $0 \%$ \\
\hline & & 2017 & 31290 & 301252 & $32.19 \%$ & $10.39 \%$ \\
\hline & & 2018 & 45005 & 290816 & $28.4 \%$ & $15.48 \%$ \\
\hline & \multirow{2}{*}{2017} & 2017 & 0 & 777106 & $0 \%$ & $0 \%$ \\
\hline & & 2018 & 44017 & 352938 & $25.22 \%$ & $12.47 \%$ \\
\hline \multirow[t]{14}{*}{ Alt-lite or I.D.W. } & \multirow[t]{5}{*}{$2006-2012$} & 2006-2012 & 0 & 227945 & $0 \%$ & $0 \%$ \\
\hline & & 2013-2015 & 3192 & 64874 & $215 \%$ & $4.92 \%$ \\
\hline & & 2016 & 5016 & 39527 & $132 \%$ & $12.69 \%$ \\
\hline & & 2017 & 6645 & 42140 & $8.94 \%$ & $15.77 \%$ \\
\hline & & 2018 & 8895 & 49748 & $6.29 \%$ & $17.88 \%$ \\
\hline & \multirow[t]{4}{*}{$2013-2015$} & 2013-2015 & 0 & 694155 & $0 \%$ & $0 \%$ \\
\hline & & 2016 & 25848 & 252962 & $57.75 \%$ & $10.22 \%$ \\
\hline & & 2017 & 33532 & 230172 & $41.91 \%$ & $14.57 \%$ \\
\hline & & 2018 & 42629 & 239116 & $29.27 \%$ & $17.83 \%$ \\
\hline & \multirow[t]{3}{*}{2016} & 2016 & 0 & 1040872 & $0 \%$ & $0 \%$ \\
\hline & & 2017 & 52610 & 480309 & $579 \%$ & $10.95 \%$ \\
\hline & & 2018 & 70905 & 454870 & $45.91 \%$ & $15.59 \%$ \\
\hline & \multirow[t]{2}{*}{2017} & 2017 & 0 & 1251674 & $0 \%$ & $0 \%$ \\
\hline & & 2018 & 74534 & 619501 & $44.1 \%$ & $123 \%$ \\
\hline \multirow[t]{14}{*}{ Media } & \multirow[t]{5}{*}{$2006-2012$} & 2006-2012 & 0 & 248214 & $0 \%$ & $0 \%$ \\
\hline & & 2013-2015 & 1136 & 50724 & $7 \%$ & $2.24 \%$ \\
\hline & & 2016 & 2331 & 27168 & $5.3 \%$ & $8.58 \%$ \\
\hline & & 2017 & 3123 & 30991 & $3.75 \%$ & $108 \%$ \\
\hline & & 2018 & 4629 & 41913 & $31 \%$ & $114 \%$ \\
\hline & \multirow[t]{4}{*}{$2013-2015$} & 2013-2015 & 0 & 637338 & $0 \%$ & $0 \%$ \\
\hline & & 2016 & 3146 & 81489 & $5.75 \%$ & $3.86 \%$ \\
\hline & & 2017 & 5159 & 86127 & $5.42 \%$ & $5.99 \%$ \\
\hline & & 2018 & 8123 & 116469 & $4.85 \%$ & $6.97 \%$ \\
\hline & \multirow{3}{*}{2016} & 2016 & 0 & 365614 & $0 \%$ & $0 \%$ \\
\hline & & 2017 & 2929 & 75512 & $2.65 \%$ & $3.88 \%$ \\
\hline & & 2018 & 5000 & 92281 & $2.79 \%$ & $5.42 \%$ \\
\hline & \multirow[t]{2}{*}{2017} & 2017 & 0 & 696297 & $0 \%$ & $0 \%$ \\
\hline & & 2018 & 7809 & 214600 & $3.86 \%$ & $3.64 \%$ \\
\hline \multirow[t]{14}{*}{ I.D.W. } & \multirow[t]{5}{*}{ 2006-2012 } & 2006-2012 & 0 & 47914 & $0 \%$ & $0 \%$ \\
\hline & & 2013-2015 & 565 & 14948 & $3.54 \%$ & $3.78 \%$ \\
\hline & & 2016 & 889 & 8745 & $2.26 \%$ & $10.17 \%$ \\
\hline & & 2017 & 1240 & 9424 & $1.64 \%$ & $13.16 \%$ \\
\hline & & 2018 & 1686 & 11322 & $1.15 \%$ & $14.89 \%$ \\
\hline & $2013-2015$ & 2013-2015 & 0 & 212122 & $0 \%$ & $0 \%$ \\
\hline & & 2016 & 4634 & 72573 & $9.54 \%$ & $6.39 \%$ \\
\hline & & 2017 & 6742 & 67199 & $7.79 \%$ & $103 \%$ \\
\hline & & 2018 & 8543 & 70677 & $5.43 \%$ & $129 \%$ \\
\hline & 2016 & 2016 & 0 & 232159 & $0 \%$ & $0 \%$ \\
\hline & & 2017 & 5942 & 98640 & $5.83 \%$ & $62 \%$ \\
\hline & & 2018 & 8711 & 97288 & $5.15 \%$ & $8.95 \%$ \\
\hline & 2017 & 2017 & 0 & 420116 & $0 \%$ & $0 \%$ \\
\hline & & 2018 & 14268 & 206053 & $7.61 \%$ & $6.92 \%$ \\
\hline
\end{tabular}


Table 6: We show absolute numbers for users infected and tracked in Sec. 6, as well as what percentage of the total number of users who watched Alt-right the number of users infected was.

\begin{tabular}{|c|c|c|c|c|c|c|}
\hline Category & Start & Year & \# Users Infected & \# Users Tracked & $\%$ of Users Alt-right & $\%$ Tracked and Infected \\
\hline \multirow[t]{14}{*}{ Alt-right to I.D.W. } & \multirow[t]{5}{*}{ 2006-2012 } & 2006-2012 & 2006-2012 & 3276 & $0 \%$ & $0 \%$ \\
\hline & & $2013-2015$ & 283 & 997 & $0.24 \%$ & $28.39 \%$ \\
\hline & & 2016 & 216 & 569 & $0.13 \%$ & $37.96 \%$ \\
\hline & & 2017 & 290 & 646 & $0.1 \%$ & $44.89 \%$ \\
\hline & & 2018 & 391 & 741 & $07 \%$ & $52.77 \%$ \\
\hline & \multirow[t]{4}{*}{$2013-2015$} & $2013-2015$ & 2006-2012 & 21578 & $0 \%$ & $0 \%$ \\
\hline & & 2016 & 1421 & 6384 & $0.72 \%$ & $22.26 \%$ \\
\hline & & 2017 & 1821 & 5691 & $0.59 \%$ & $32 \%$ \\
\hline & & 2018 & 2565 & 5922 & $0.43 \%$ & $43.31 \%$ \\
\hline & \multirow{3}{*}{2016} & 2016 & 2006-2012 & 41385 & $0 \%$ & $0 \%$ \\
\hline & & 2017 & 4142 & 15752 & $1.17 \%$ & $26.3 \%$ \\
\hline & & 2018 & 5511 & 15426 & $0.87 \%$ & $35.73 \%$ \\
\hline & \multirow[t]{2}{*}{2017} & 2017 & 2006-2012 & 69241 & $0 \%$ & $0 \%$ \\
\hline & & 2018 & 9024 & 29987 & $1.31 \%$ & $309 \%$ \\
\hline \multirow{14}{*}{ Alt-right to Alt-lite } & \multirow{5}{*}{$2006-2012$} & $2006-2012$ & 2006-2012 & 3276 & $0 \%$ & $0 \%$ \\
\hline & & $2013-2015$ & 465 & 997 & $0.29 \%$ & $46.64 \%$ \\
\hline & & 2016 & 399 & 569 & $0.12 \%$ & $70.12 \%$ \\
\hline & & 2017 & 410 & 646 & $0.1 \%$ & $63.47 \%$ \\
\hline & & 2018 & 407 & 741 & $06 \%$ & $54.93 \%$ \\
\hline & \multirow[t]{4}{*}{$2013-2015$} & $2013-2015$ & 2006-2012 & 21578 & $0 \%$ & $0 \%$ \\
\hline & & 2016 & 3047 & 6384 & $0.87 \%$ & $47.73 \%$ \\
\hline & & 2017 & 2962 & 5691 & $0.64 \%$ & $525 \%$ \\
\hline & & 2018 & 2884 & 5922 & $0.43 \%$ & $48.7 \%$ \\
\hline & \multirow[t]{3}{*}{2016} & 2016 & 2006-2012 & 41385 & $0 \%$ & $0 \%$ \\
\hline & & 2017 & 7696 & 15752 & $1.49 \%$ & $48.86 \%$ \\
\hline & & 2018 & 7089 & 15426 & $0.98 \%$ & $45.95 \%$ \\
\hline & \multirow[t]{2}{*}{2017} & 2017 & 2006-2012 & 69241 & $0 \%$ & $0 \%$ \\
\hline & & 2018 & 13435 & 29987 & $1.79 \%$ & $44.8 \%$ \\
\hline \multirow[t]{14}{*}{ Alt-right to Media } & \multirow[t]{5}{*}{ 2006-2012 } & $2006-2012$ & 0.0 & 3276.0 & $0.0 \%$ & $0.0 \%$ \\
\hline & & $2013-2015$ & 407.0 & 997.0 & $0.21 \%$ & $40.82 \%$ \\
\hline & & 2016 & 152.0 & 569.0 & $0.12 \%$ & $26.71 \%$ \\
\hline & & 2017 & 225.0 & 646.0 & $0.09 \%$ & $34.83 \%$ \\
\hline & & 2018 & 406.0 & 741.0 & $0.05 \%$ & $54.79 \%$ \\
\hline & \multirow{4}{*}{$2013-2015$} & $2013-2015$ & 0.0 & 21578.0 & $0.0 \%$ & $0.0 \%$ \\
\hline & & 2016 & 1043.0 & 6384.0 & $0.6 \%$ & $16.34 \%$ \\
\hline & & 2017 & 1504.0 & 5691.0 & $0.49 \%$ & $26.43 \%$ \\
\hline & & 2018 & 2872.0 & 5922.0 & $0.29 \%$ & $48.5 \%$ \\
\hline & \multirow[t]{3}{*}{2016} & 2016 & 0.0 & 41385.0 & $0.0 \%$ & $0.0 \%$ \\
\hline & & 2017 & 4787.0 & 15752.0 & $1.41 \%$ & $30.39 \%$ \\
\hline & & 2018 & 8639.0 & 15426.0 & $0.84 \%$ & $56.0 \%$ \\
\hline & \multirow[t]{2}{*}{2017} & 2017 & 0.0 & 69241.0 & $0.0 \%$ & $0.0 \%$ \\
\hline & & 2018 & 16269.0 & 29987.0 & $1.46 \%$ & $54.25 \%$ \\
\hline
\end{tabular}


Table 7: For the three communities, we list all the websites analysed in this paper (part 1).

\begin{tabular}{|c|c|c|c|c|c|c|}
\hline & Alt-right channels & Step & Alt-lite channels & Step & I.D.W. channels & Step \\
\hline 0 & AltRight.com & 1 & America First with Nicholas J Fuentes & 1 & Ben Shapiro & 1 \\
\hline 1 & AmRen Podcasts & 1 & Andy Warski & 1 & Bret Weinstein & 1 \\
\hline 2 & AmRenVideos & 1 & Blonde in the Belly of the Beast & 1 & Gad Saad & 1 \\
\hline 3 & Ayla Stewart Wife With A Purpose & 1 & Brittany Pettibone & 1 & JRE Clips & 1 \\
\hline 4 & Baked Alaska 2 & 1 & Computing Forever & 1 & Jordan B Peterson Clips & 1 \\
\hline 5 & Black Pigeon Speaks & 1 & Gavin McInnes & 1 & JordanPetersonVideos & 1 \\
\hline 6 & Bre Faucheux & 1 & Laura Loomer & 1 & Lindsay Shepherd & 1 \\
\hline 7 & CounterCurrentsTV & 1 & Lauren Chen & 1 & Matt Christiansen & 1 \\
\hline 8 & Darkstream & 1 & Lauren Southern & 1 & Owen Benjamin & 1 \\
\hline 9 & Faith J Goldy & 1 & MILO & 1 & Owen Benjamin Clips & 1 \\
\hline 10 & James Allsup & 1 & Mike Cernovich & 1 & PowerfulJRE & 1 \\
\hline 11 & Jason Kessler & 1 & Nick Fuentes Clips & 1 & Rebel Wisdom & 1 \\
\hline 12 & Jean-François Gariépy & 1 & No Bullshit & 1 & Sam Harris & 1 \\
\hline 13 & Johnny Monoxide & 1 & No Bullshit 2 & 1 & SargonofAkkad100 & 1 \\
\hline 14 & MW Live & 1 & Paul Joseph Watson & 1 & The Rubin Report & 1 \\
\hline 15 & Matt Forney & 1 & Rebel Canada & 1 & joerogandotnet & 1 \\
\hline 16 & MillennialWoes & 1 & Rebel Edge & 1 & 1791 & 2 \\
\hline 17 & NPI / Radix & 1 & Rebel Media & 1 & American Justice & 2 \\
\hline 18 & Red Ice TV & 1 & Stefan Molyneux & 1 & Atheist Foundation of Australia Inc & 2 \\
\hline 19 & Staying Woke & 1 & StevenCrowder & 1 & AynRandInstitute & 2 \\
\hline 20 & The Golden One & 1 & Styxhexenhammer666 & 1 & Ben Shapiro Thug Life & 2 \\
\hline 21 & The Reality Calls Show & 1 & The Thinkery & 1 & Benjamin A Boyce & 2 \\
\hline 22 & Traditionalist Worker Party & 1 & Vee & 1 & Brother Nathanael & 2 \\
\hline 23 & Voxiversity & 1 & 6oodfella & 2 & CISAus & 2 \\
\hline 24 & augustussolinvictus & 1 & A1Cvenom & 2 & Clash of Ideas & 2 \\
\hline 25 & iambakedalaska & 1 & AIU-Resurrection & 2 & Conversations with Bill Kristol & 2 \\
\hline 26 & Alt Right & 2 & AltRight Truth & 2 & Crysta & 2 \\
\hline 27 & Alt-Right Tankie & 2 & AustralianNeoCon 1 & 2 & Desi-Rae Thinking & 2 \\
\hline 28 & American Pride & 2 & BlazeTV & 2 & Douglas Murray Archive & 2 \\
\hline 29 & American Pride 2 & 2 & Brave New World & 2 & Enlightainment & 2 \\
\hline 30 & ArktosOnline & 2 & Bull Brand & 2 & Essential Truth & 2 \\
\hline 31 & Augustus Invictus for United States Senate & 2 & Carpe Donktum & 2 & Freedom Speaks & 2 \\
\hline 32 & AustralianRealist & 2 & Christopher Anderson & 2 & Glenn Beck & 2 \\
\hline 33 & Be Open MInded & 2 & Daily Caller & 2 & Gravitahn & 2 \\
\hline 34 & BigCatKayla Livestreams & 2 & DailyCallerVideo & 2 & Informative & 2 \\
\hline 35 & Charles Zeiger & 2 & DailyKenn & 2 & Jordan Peterson Fan Channel & 2 \\
\hline 36 & Corpus Mentis & 2 & Dinesh D’Souza & 2 & Liberty us & 2 \\
\hline 37 & Dismantle The Matrix & 2 & DoctorRandomercam & 2 & MG & 2 \\
\hline 38 & Dissident View & 2 & Domination Station & 2 & Maximilien Robespierre & 2 \\
\hline 39 & Engländer & 2 & Harrison Hill Smith & 2 & MeaningofLife.tv & 2 \\
\hline 40 & Jan Kerkoff & 2 & Jacob Wohl & 2 & Mike Nayna & 2 \\
\hline 41 & Mark Collett & 2 & Kelly Day & 2 & Motte \& Bailey & 2 \\
\hline 42 & Matthew North & 2 & Leo Stratton & 2 & MrAndsn & 2 \\
\hline 43 & Nacionalista Blanco del SoCal & 2 & Liberty Machine News & 2 & Notes For Space Cadets & 2 \\
\hline 44 & Nationalist Media Network & 2 & Luke Ford & 2 & Pangburn & 2 \\
\hline 45 & No White Guilt & 2 & Luke Ford Livestreams & 2 & PhilosophyInsights & 2 \\
\hline 46 & Patrick Slattery & 2 & Make Cringe Great Again & 2 & Pragmatic Entertainment & 2 \\
\hline 47 & Real McGoy & 2 & News2Share & 2 & ReasonTV & 2 \\
\hline 48 & Revcon Media & 2 & On The Offensive & 2 & Savage Facts & 2 \\
\hline 49 & Stand Up Europe & 2 & Oppressed Media & 2 & The Daily Truth & 2 \\
\hline 50 & Steve Trueblue & 2 & Revenge Of The Cis & 2 & The Free Speech Club & 2 \\
\hline 51 & The Alternative Hypothesis & 2 & RobinHoodUKIP & 2 & The Heritage Foundation & 2 \\
\hline 52 & The Great Dolemite & 2 & SJW CRINGE MACHINE & 2 & The New Criterion & 2 \\
\hline 53 & The James Delingpole Channel & 2 & SJWCentral & 2 & The Pondering Primate & 2 \\
\hline 54 & The Last Stand & 2 & Semiogogue & 2 & The Unplugged Observer & 2 \\
\hline 55 & The Rational Rise & 2 & Social Justice Fails & 2 & TheArchangel911 & 2 \\
\hline 56 & TheArmenianNation & 2 & The Fallen State & 2 & TheAtlasSociety & 2 \\
\hline 57 & This is Europa & 2 & The Glass Blind Spot & 2 & Transliminal & 2 \\
\hline
\end{tabular}


Table 8: For the three communities, we list all the websites analysed in this paper (part 2).

\begin{tabular}{|c|c|c|c|c|c|c|}
\hline & Alt-right channels & Step & Alt-lite channels & Step & I.D.W. channels & Step \\
\hline 58 & ThuleanPerspective & 2 & The Hateful Gaels & 2 & Trigger Happy Media & 2 \\
\hline 59 & Traditionalist Youth Network & 2 & The Iconoclast & 2 & VikNand & 2 \\
\hline 60 & Truth Against The World & 2 & TheSchillingShow & 2 & Washington Watch & 2 \\
\hline 61 & WhiteRabbitRadioTV & 2 & Tipping Point With Liz Wheeler on OAN & 2 & WisdomTalks & 2 \\
\hline 62 & andy nowicki & 2 & Tommy Robinson & 2 & YAFTV & 2 \\
\hline 63 & eliharman & 2 & Tree Of Logic & 2 & ZIEeICoZ & 2 \\
\hline 64 & jackburton2009 & 2 & UNITE AMERICA FIRST & 2 & ZeroFox Given & 2 \\
\hline 65 & nightmarefuel & 2 & Western Man & 2 & battleofideas & 2 \\
\hline 66 & 14 Sacred Words & 3 & Zach Hing & 2 & bloggingheads.tv & 2 \\
\hline 67 & Awakened Saxon & 3 & grapjas60 & 2 & bmdavll & 2 \\
\hline 68 & Borzoi Boskovic & 3 & hOrnsticles3 & 2 & successcouncil & 2 \\
\hline 69 & Danny 1488 & 3 & ramzpaul & 2 & tmcleanful & 2 \\
\hline 70 & InvincibleNumanist & 3 & theovonk & 2 & wikileaksplus & 2 \\
\hline 71 & LaughingMan0X & 3 & theturningpointusa & 2 & xUnlimitedMagz & 2 \\
\hline 72 & Laura Towler & 3 & thkelly 67 & 2 & ybrook & 2 \\
\hline 73 & LibertarianRealist2 & 3 & Actual Justice Warrior & 3 & AgatanFoundation & 3 \\
\hline 74 & Little Revolution & 3 & AllNationsParty & 3 & Bite-sized Philosophy & 3 \\
\hline 75 & Marie Cachet & 3 & Alt Hype Streams & 3 & CoolHardLogic & 3 \\
\hline 76 & Morrakiu & 3 & Aydin Paladin & 3 & Davie Addison & 3 \\
\hline 77 & NeatoBurrito Productions & 3 & Beacom Of Light & 3 & Dose of Truth & 3 \\
\hline 78 & NewEuropeANP & 3 & Bearing & 3 & DronetekPolitics & 3 \\
\hline 79 & OnlineWipe & 3 & Count Dankula & 3 & Galactic Bubble Productions & 3 \\
\hline 80 & Oswald Spengler & 3 & Dangerfield & 3 & HowTheWorldWorks & 3 \\
\hline 81 & Prince of Zimbabwe & 3 & Demirep & 3 & ManOfAllCreation & 3 \\
\hline 82 & Serp Kerp & 3 & Dr. Steve Turley & 3 & PragerUniversity & 3 \\
\hline 83 & TRS Radio & 3 & IRmep Stream & 3 & Rekt Idiots & 3 \\
\hline 84 & The Leftovers & 3 & Jericho Green & 3 & Sinatra_Says & 3 \\
\hline 85 & The Lion & 3 & John Ward & 3 & Sorting Myself Out & 3 \\
\hline 86 & The Revolutionary Conservative & 3 & JustInformed Talk & 3 & The Andrew Klavan Show & 3 \\
\hline 87 & VertigoPolitix & 3 & Liberty Hangout & 3 & The Daily Wire & 3 \\
\hline 88 & & & MR. OBVIOUS & 3 & The Heartland Institute & 3 \\
\hline 89 & & & MarkDice & 3 & The Propertarian Institute & 3 \\
\hline 90 & & & MichelleRempel & 3 & Timcast & 3 \\
\hline 91 & & & Mister Metokur & 3 & & \\
\hline 92 & & & NateTalksToYou & 3 & & \\
\hline 93 & & & OneTruth4Life & 3 & & \\
\hline 94 & & & ProductiehuisEU & 3 & & \\
\hline 95 & & & Reverend Simon Sideways & 3 & & \\
\hline 96 & & & Sanity 4 Sweden & 3 & & \\
\hline 97 & & & Sargon of Akkad Live & 3 & & \\
\hline 98 & & & SkidRowRadio & 3 & & \\
\hline 99 & & & Slightly Offens*ve & 3 & & \\
\hline 100 & & & Tea Clips & 3 & & \\
\hline 101 & & & The Amazing Lucas & 3 & & \\
\hline 102 & & & The Weekly Sweat & 3 & & \\
\hline 103 & & & TheBechtloff & 3 & & \\
\hline 104 & & & TheIncredibleSaltMine & 3 & & \\
\hline 105 & & & Toad McKinley & 3 & & \\
\hline 106 & & & TokenLibertarianGirl & 3 & & \\
\hline 107 & & & Undoomed & 3 & & \\
\hline 108 & & & Vincent James of The Red Elephants & 3 & & \\
\hline 109 & & & ataxin & 3 & & \\
\hline 110 & & & brianoflondon & 3 & & \\
\hline 111 & & & jaydyer & 3 & & \\
\hline 112 & & & libertydollshouse & 3 & & \\
\hline 113 & & & patcondell & 3 & & \\
\hline
\end{tabular}


Table 9: Media channels.

\begin{tabular}{|c|c|c|c|c|c|}
\hline & Left & Center & Left-Center & Right-Center & Right \\
\hline 0 & cosmopolitan & big think & (the)atlantic & forbes & american enterprise institute \\
\hline 1 & democracy now & c-span & business insider & gulf news & judicial watch \\
\hline 3 & good magazine & financial times & engadget & new york post & pj media \\
\hline 4 & gq magazine & harvard business review & feminist frequency & ntd.tv (new tang dynasty) & project veritas \\
\hline 5 & huffington post (huffpost) & investopedia & glamour magazine & russia insider & ron paul liberty report \\
\hline 7 & merry jane & mental floss & global news & & \\
\hline 8 & new york magazine & military.com & hollywood reporter & & \\
\hline 9 & new yorker & recode & la times & & \\
\hline 10 & people magazine & relevant magazine & lifehacker & & \\
\hline 11 & slate & the economist & new york daily news & & \\
\hline 12 & uproxx & the indian express & rolling stone & & \\
\hline 13 & upworthy & today i found out & san francisco globe & & \\
\hline 15 & vox & world economic forum & scroll.in & & \\
\hline 16 & & & sky news & & \\
\hline 17 & & & techcrunch & & \\
\hline 18 & & & the guardian & & \\
\hline 19 & & & the verge & & \\
\hline 20 & & & vice news & & \\
\hline 21 & & & washington post & & \\
\hline 22 & & & wired magazine & & \\
\hline 23 & & & yahoo news & & \\
\hline
\end{tabular}




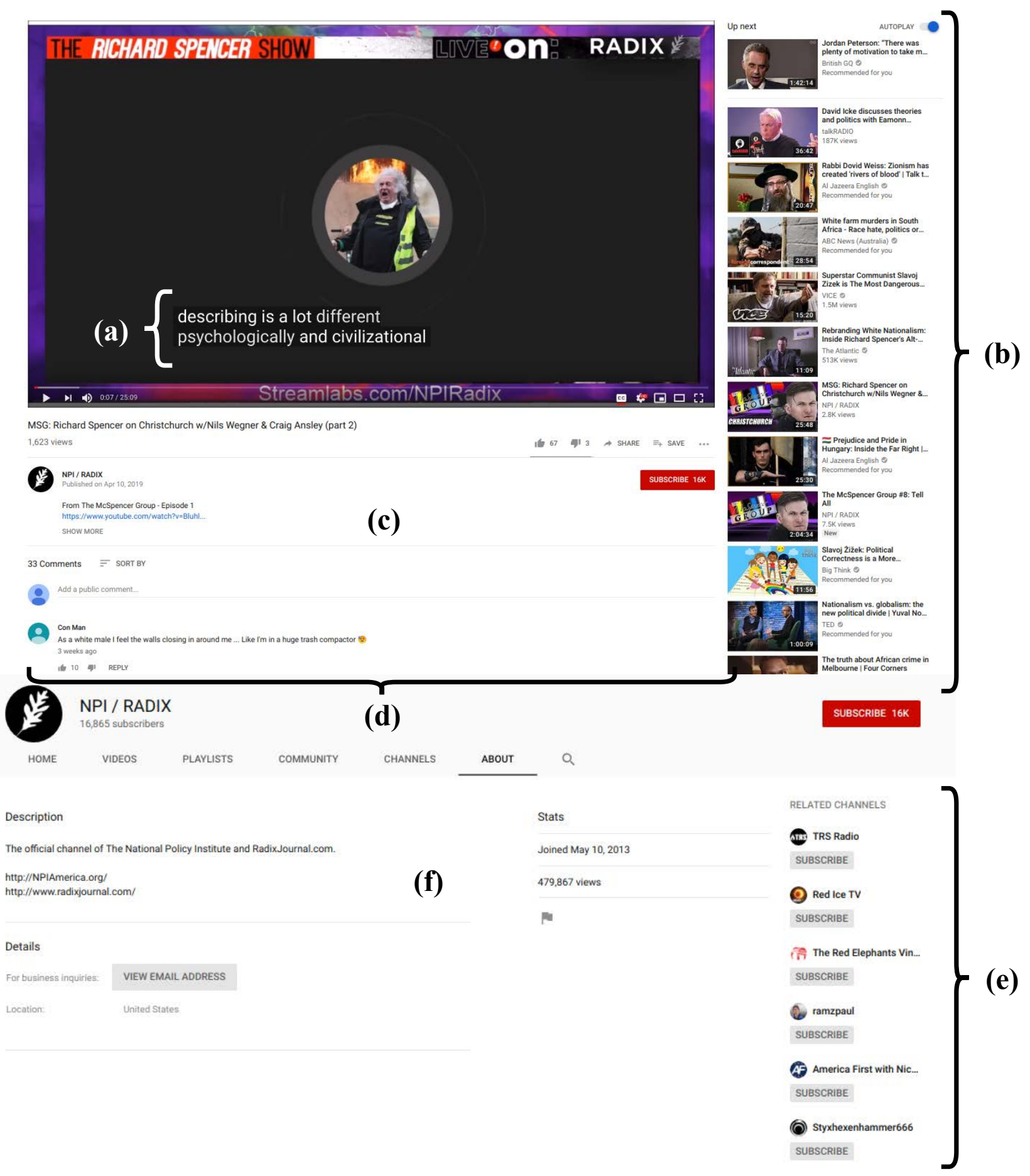

Figure 9: Overview of the elements we collected: (a) video captions, when available, (b) video recommendations, (c) video description and metadata, (d) comments, (e) channel recommendations, and (f) video metadata. 